\title{
Article
}

\section{Stability of stochastic 2D Navier-Stokes equations with memory and Poisson jumps}

\section{Diem Dang Huan}

Than Nhan Trung High School, Bacgiang Agriculture and Forestry University, Bacgiang 21000, Vietnam.; diemdanghuan@gmail.com

Received: 28 March 2020; Accepted: 11 November 2020; Published: 30 November 2020.

Abstract: The objective of this paper is to study the stability of the weak solutions of stochastic 2D Navier-Stokes equations with memory and Poisson jumps. The asymptotic stability of the stochastic Navier-Stoke equation as a semilinear stochastic evolution equation in Hilbert spaces is obtained in both mean square and almost sure senses. Our results can extend and improve some existing ones.

Keywords: Stochastic Navier-Stokes equations, delays, Lévy noise.

MSC: 60H15, 76D05.

\section{Introduction}

I

$\mathrm{n}$ this paper, we will investigate the stability of the weak solutions of stochastic 2D Navier-Stokes equations with memory and Poisson jumps of the form:

$$
\left\{\begin{aligned}
& d X= {[v \triangle X-\langle X, \nabla\rangle X-\nabla p+f(x)+g(X(t-\rho(t)))] d t+\sigma(t, X(t-\delta(t))) d W(t) } \\
&+\int_{Z} k(t, X(t-\gamma(t)), z) \widetilde{\eta}(d t, d z) \\
& \operatorname{div} X=0 \text { in }(0,+\infty) \times D, \quad X(t, x)=0 \quad \text { on } \quad(0,+\infty) \times \partial D \\
& X(0, x)=X_{0}(x), \quad X(t, x)=\phi(t, x), \text { for } x \in D, \text { and } t \in[-r, 0], \text { with } r>0
\end{aligned}\right.
$$

where $X$ is the velocity field of the fluid, $v>0$ is the kinematic viscosity, $p$ is the associated hydrostatic pressure, $f$ is a non-delayed external force field, $g(X(t-\rho(t)))$ is another external force field with delay, $\sigma(t, X(t-\delta(t))) d W(t)+\int_{Z} k(t, X(t-\gamma(t)), z) \widetilde{\eta}(d t, d z)$ is a random external force field with delays, where $W$ is an infinite dimensional Wiener process and $\widetilde{\eta}$ is a compensated time homogeneous Poisson random measure, $\rho, \delta, \gamma: \mathbb{R}^{+} \rightarrow[0, r]$ are continuous functions, $D$ is a regular open bounded domain of $\mathbb{R}^{2}$ with boundary $\partial D$, $X_{0}$ is the initial velocity, $\phi$ is the initial datum in the interval $[-r, 0]$.

Navier-Stokes equations are the fundamental model of the fluid mechanics and turbulence. These equations have been the object of numerous works (see, for instance [1,2] and the references therein), even with unbounded domains (see [2,3]) since the first paper of Leray was published in 1933 [4]. Beside, noise or stochastic perturbation is unavoidable and omnipresent in nature as well as in man-made systems. Therefore, it is of great significance to import the stochastic effects into the investigation of Navier-Stokes equations. To our best knowledge, the theory of stochastic Navier-Stokes equations apparently has its roots in the 1959 edition of the Landau and Lifshitz [5] and the first work on the stochastic Navier-Stokes equations written from the mathematical point of view is the paper [6]. We mention here the works ([7-10] and the references cited therein) concerning the two-dimensional (2D in short) stochastic Navier-Stokes equation.

Partial differential equations with memory (e.g. delay) have attracted great interest due to their applications in describing many sophisticated dynamical systems in physics, chemistry, biology, economics and social sciences. On this matter, we refer the reader to [11-16] and the references therein. In the past few years, many papers have studied the Navier-Stokes equations with a forcing term which contains some hereditary characteristics (see, for instance [17-19] and the references therein). In addition, the long-time behavior and exponential stability of the Navier-Stokes equations is an interesting and challenging problem, since it can provide useful information on the future evolution of the system (see, [20,21]). On the exponential behavior for stochastic 2D Navier-Stokes equations with variable delay, we refer the reader to [22-24], and 
recently, in [25] and [26], the authors investigated the asymptotic behavior of solutions of stochastic evolution equations for second grade fluids and non-Newtonian fluids, respectively.

On the other hand, the world is more complicated and models which are allowed to have jumps - both big and small - are desirable. Hence, it is necessary and important to study stochastic systems with Poisson jumps or Lévy processes (see, for instance [27-31] and the references therein). In recent years, the stochastic 2D(3D) Navier-Stokes equations with Lévy noise has attracted much attention of researchers. To be more precise, in [32], Motyl considered the existence of solutions, in a probabilistic sense, to the stochastically driven, viscous, incompressible Navier-Stokes equations driven by the Lévy noise consisting of the compensated time homogeneous Poisson random measure and the Wiener process in two and three spatial dimensions, Dong et al., [33] discussed the existence of stationary weak solutions of stochastic 3D Navier-Stokes equations involving jumps and compared the Galerkin stationary probability measures for the case driven by Lévy noise and the one driven by Wiener process, and in [34], by using an abstract setting, Brzeźniak et al. studied the existence and uniqueness of the solution of an abstract nonlinear equation driven by a multiplicative noise of Lévy type. More specifically, just recently, Taniguchi [35] obtained the existence and exponential stability of energy solutions to 2D stochastic functional Navier-Stokes equation perturbed by the Lévy process. However, to prove the exponential stability results in [35], the author have imposed that the delay function $(\rho(t))$ appears only in the non-random external force and furthermore this delay function have required a strong assumption, i.e. $\rho(t)$ is differentiable and satisfies $0 \leq \rho^{\prime}(t)<M_{*}<1$, where $M_{*}>0$. Thus, we will make the first attempt to establish some results for more general forcing terms and relax this restriction. Under some suitable assumptions, by using the Itô formula for jumps and Burkholder-Davis-Gundy inequalities in stochastic analysis, we establish the weak solution to (1) converges to the stationary solution of its stationary versions exponentially stable in the mean square. Further, by establishing a lemma for compensated Poisson random measures (Lemma 3), we give the result that the weak solution to (1) converges to the stationary solution of its stationary version almost surely exponentially. Assumptions given in our article do not require the monotone decreasing behaviour of the delays and satisfying $0 \leq \rho^{\prime}(t), \delta^{\prime}(t), \gamma^{\prime}(t)<1$. Therefore, the current paper can be regarded as the extension of the work of Caraballo and Real [20] to the stochastic settings, simultaneously extend and improve the one of Taniguchi [35] (where the random external force field does not include delays and the external force field contains delay but the memory function $\rho(t)$ is a differentiable function) as well as the asymptotic behavior results published in [7] (where the external force fields does not contain delays and the stochastic Navier-Stokes equation without non-Gaussian Lévy noise perturbation) and the papers announced by Chen [22], Wan and Zhou [23] (where the random external force field does not contain discontinuous multiplicative noise).

The remainder of this paper is organized as follows: In Section 2, we briefly present some basic notations, preliminaries. The main results in Section 3 are devoted to studying the asymptotic behavior for the weak solutions of the system (1) with their proofs.

\section{Preliminaries}

In this section, we introduce notations and preliminary results need to establish our results. For more details on this section, we refer the reader to $[2,8,36-38]$.

We first introduce the following function spaces, which are usual in the study of Navier-Stokes equations:

$$
\mathcal{V}:=\left\{u \in C_{0}^{\infty}\left(D, \mathbb{R}^{2}\right): \operatorname{div} u=0\right\} .
$$

$\mathbb{H}:=$ the closure of $\mathcal{V}$ in $L^{2}\left(D, \mathbb{R}^{2}\right)$ with the norm $|u|=(u, u)^{\frac{1}{2}}$, where for $u, v \in L^{2}\left(D, \mathbb{R}^{2}\right)$,

$$
(u, v)=\sum_{j=1}^{2} \int_{D} u_{j}(x) v_{j}(x) d x .
$$

$\mathbb{V}:=$ the closure of $\mathcal{V}$ in $\mathbb{H}_{0}^{1}\left(D, \mathbb{R}^{2}\right)$ with the norm $\|u\|=((u, u))^{\frac{1}{2}}$, where for $u, v \in \mathbb{H}_{0}^{1}\left(D, \mathbb{R}^{2}\right)$,

$$
((u, v))=\sum_{i, j=1}^{2} \int_{D} \frac{\partial u_{i}}{\partial x_{j}} \frac{\partial v_{i}}{\partial x_{j}} d x
$$


It follows that $\mathbb{H}$ and $\mathbb{V}$ are separable Hilbert spaces with associated inner products $(\cdot, \cdot)$ and $((\cdot, \cdot))$ and the following is satisfied

$$
\mathbb{V} \subset \mathbb{H} \equiv \mathbb{H}^{*} \subset \mathbb{V}^{*},
$$

where injections are dense, continuous, and compact; $\mathbb{H}^{*}$ and $\mathbb{V}^{*}$ stand for the topological dual of $\mathbb{H}$ and $\mathbb{V}$, respectively.

Now, let $P_{\mathbb{H}}$ be an orthogonal projector from $L^{2}\left(D, \mathbb{R}^{2}\right)$ onto $\mathbb{H}$. We define the operator $A: L^{2}\left(D, \mathbb{R}^{2}\right) \rightarrow \mathbb{H}$ by $A u=-P_{\mathbb{H}} \triangle u$ and $B: \mathbb{V} \times \mathbb{V} \rightarrow \mathbb{V}^{*}$ by $\langle B(u, v), w\rangle=b(u, v, w), \forall u, v, w \in \mathbb{V}$, where $\langle\cdot, \cdot\rangle$ denotes the duality $\left\langle\mathbb{V}^{*}, \mathbb{V}\right\rangle$ and $b$ is the trilinear form defined by

$$
b(u, v, w)=\sum_{i, j=1}^{2} \int_{D} u^{j}(x) \frac{\partial v^{j}}{\partial x_{i}} w^{j}(x) d x .
$$

We also set $B(u)=B(u, u), \forall u \in \mathbb{V}$.

Furthermore, we shall need some properties of the trilinear form $b$, and we list below the ones that we will used later on (see, [38]),

$$
\begin{aligned}
& |b(u, v, w)| \leq C_{1}|u|^{\frac{1}{2}}\|u\|^{\frac{1}{2}}\|v\||w|^{\frac{1}{2}}\|w\|^{\frac{1}{2}}, \quad \forall u, v, w \in \mathbb{V}, \\
& b(u, v, v)=0, \quad \forall u, v \in \mathbb{V}, \\
& b(u, u, v-u)-b(v, v, v-u)=-b(v-u, u, v-u), \quad \forall u, v \in \mathbb{V},
\end{aligned}
$$

where $C_{1}>0$ is an appropriate constant which depends on the regular open domain $D$ (see [39]).

Let $(\Omega, \mathcal{F}, \mathbf{P})$ be a complete probability space equipped with some filtration $\left(\mathcal{F}_{t}\right)_{t \geqslant 0}$ satisfying the usual conditions (i.e., it is right continuous and $\mathcal{F}_{0}$ contains all P-null sets).

With the symbol $\{W(t)\}_{t \geq 0}$, we denote a $\mathbb{K}$-valued $\left(\mathcal{F}_{t}\right)_{t \geqslant 0}$-Wiener process defined on the probability space $(\Omega, \mathcal{F}, \mathbf{P})$ with covariance operator $Q$, i.e.

$$
\mathbf{E}\langle W(t), a\rangle_{\mathbb{K}}\langle W(s), b\rangle_{\mathbb{K}}=\min \{t, s\}\langle Q a, b\rangle_{\mathbb{K}}, \quad \forall a, b \in \mathbb{K},
$$

where $Q$ is a positive, self-adjoint, trace class operator on $\mathbb{K}$. In particular, we call such $\{W(t)\}_{t \geq 0}$ a $\mathbb{K}$-valued $Q$-Wiener process relative to $\left(\mathcal{F}_{t}\right)_{t \geqslant 0}$. We assume that there exist a complete orthonormal system $\left\{e_{n}\right\}_{n \in \mathbb{N}}$ in $\mathbb{K}$, a bounded sequence of nonnegative real number $\left\{\lambda_{n}\right\}_{n \in \mathbb{N}}$ such that $Q e_{n}=\lambda_{n} e_{n}, \quad n=1,2,3, \ldots$, and a sequence $\left\{\beta_{n}\right\}_{n \geq 1}$ of independent standard Brownian motions such that

$$
\langle W(t), e\rangle_{\mathbb{K}}=\left\langle\sum_{n=1}^{\infty} \sqrt{\lambda_{n}} e_{n} \beta_{n}, e\right\rangle_{\mathbb{K}^{\prime}} \quad t \geq 0, \quad e \in \mathbb{K}
$$

and let $\mathcal{F}_{t}=\sigma\{W(s): 0 \leq s \leq t\}$ be the $\sigma$-algebra generated by $W$.

In order to define stochastic integrals with respect to the $Q$-Wiener process $W(t)$, we introduce the subspace $\mathbb{K}_{0}=Q^{\frac{1}{2}} \mathbb{K}$ of $\mathbb{K}$, which endowed with the inner product,

$$
\langle a, b\rangle_{\mathbb{K}_{0}}=\left\langle Q^{-\frac{1}{2}} a, Q^{-\frac{1}{2}} b\right\rangle_{\mathbb{K}}, \quad \forall a, b \in \mathbb{K}_{0}
$$

is a Hilbert space. Let $\mathcal{L}_{2}^{0}=\mathcal{L}_{2}\left(\mathbb{K}_{0} ; \mathbb{H}\right)$ denote the space of all Hilbert-Schmidt operators from $\mathbb{K}_{0}$ into $\mathbb{H}$. It turns out to be a separable Hilbert space, equipped with the norm

$$
\|\psi\|_{\mathcal{L}_{2}^{0}}^{2}=\operatorname{tr}\left(\left(\psi Q^{\frac{1}{2}}\right)\left(\psi Q^{\frac{1}{2}}\right)^{*}\right),
$$

for any $\psi \in \mathcal{L}_{2}^{0}$. Obviously, for any bounded operators $\psi \in \mathcal{L}(\mathbb{K} ; \mathbb{H})$ - the set of all linear bounded operators from $\mathbb{K}$ into $\mathbb{H}$, this norm reduces to

$$
\|\psi\|_{\mathcal{L}_{2}^{0}}^{2}=\operatorname{tr}\left(\psi Q \psi^{*}\right)=\sum_{n=1}^{\infty}\left|\sqrt{\lambda_{n}} \psi e_{n}\right|^{2} .
$$


Let $\Phi:(0, \infty) \rightarrow \mathcal{L}_{2}^{0}$ be a predictable, $\mathcal{F}_{t}$-adapted process such that

$$
\int_{0}^{t} \mathbf{E}\|\Phi(s)\|_{\mathcal{L}_{2}^{0}}^{2} d s<\infty, \quad t>0
$$

Then, we can define the $\mathbb{H}$-valued stochastic integral $\int_{0}^{t} \Phi(s) d W(s)$ (which is a continuous square-integrable martingale) of $\Phi$ with respect to the $\mathcal{F}_{t}$-valued $Q$-Wiener process $W(t)$ by

$$
\left\langle\int_{0}^{t} \Phi(s) d W(s), e\right\rangle:=\sum_{n=1}^{\infty} \int_{0}^{t}\left\langle\Phi(s) \sqrt{\lambda_{n}} e_{n}, e\right\rangle d \beta_{n}(s)
$$

for any $e \in \mathbb{H}$ using the Itô integral with respect to $\beta_{n}(s)$. For the construction, we can refer to Da Prato and Zabczyk [8].

Let $L=\left(L_{t}\right)_{t \geq 0}$ is a $\mathbb{K}$-valued Lévy processes such that $L$ has stationary and independent increments, is stochastically continuous and satisfies $L_{0}=0$ almost surely. Let $p(t), t \geq 0$ be the law of $L_{t}$, then $(p(t))_{t \geq 0}$ is a weakly continuous convolution semigroup of probability measures on $\mathbb{K}$. We have the Lévy-Khinchin formula [36] which yields for all $t \geq 0, x \in \mathbb{K}$,

$$
\mathbf{E}\left(e^{i\left\langle x, L_{t}\right\rangle_{\mathbb{K}}}\right)=e^{t \zeta(x)},
$$

where

$$
\zeta(x):=\exp \left\{i\langle a, x\rangle_{\mathbb{K}}-\frac{1}{2}\langle Q x, x\rangle_{\mathbb{K}}+\int_{\mathbb{K}-\{0\}}\left[e^{i\langle x, y\rangle_{\mathbb{K}}}-1-\chi_{\left\{|y|_{\mathbb{K}}<1\right\}}(y) i\langle x, y\rangle_{\mathbb{K}}\right] \lambda(d y)\right\}
$$

where $a \in \mathbb{K}$ and $\lambda$ is a Lévy measure or a jump intensity measure of $L$ on $\mathbb{K}-\{0\}$, i.e., $\int_{\mathbb{K}-\{0\}} \min \left(|y|_{\mathbb{K}}^{2}, 1\right) \lambda(d y)<\infty ; \chi_{Z}$ denotes the characteristic function on set $Z \subset \mathbb{K}$; the triple $(a, Q, \lambda)$ is the characteristics of $L$ and the mapping $\zeta$ is the characteristic exponent of $L$. We can also define the Lévy measure on the whole of $\mathbb{K}$ via the assignment $\lambda(\{0\})=0$.

Now, we shall write $\triangle L_{t}:=L_{t}-L_{t-}, \forall t \geq 0$, where $L_{t-}:=\lim _{s \uparrow t} L_{s}$. Then, almost surely for any $Z \in \mathcal{B}(\mathbb{K}-\{0\})$, which denotes the Borel $\sigma$-field of $(\mathbb{K}-\{0\})$ and with $0 \notin$ the closure of $Z$, we get a counting Poisson random measure $\eta$ on $(\mathbb{K}-\{0\})$ :

$$
\eta(t, Z)=\#\left\{0 \leq s \leq t, \triangle L_{s} \in Z\right\}<\infty, \quad t \geq 0 .
$$

Let

$$
\widetilde{\eta}(t, d y):=\eta(t, d y)-\lambda(d y) t
$$

be the compensated Poisson measure that is independent of $W(t)$.

Let $\lambda_{Z}$ denotes the restriction of the measure $\lambda$ to $Z$, still denoted by $\lambda$, such that $\lambda$ is finite on $Z$. Denote by $\mathcal{P}^{2}([0, T] \times Z ; \mathbb{H})$ the space of all predictable mappings $k:[0, T] \times Z \rightarrow \mathbb{H}$ for which

$$
\int_{0}^{T} \int_{Z} \mathbf{E}|k(t, y)|_{\mathbb{H}}^{2} \lambda(d y) d t<\infty
$$

We may then define the $\mathbb{H}$-valued stochastic integral

$$
\int_{0}^{T} \int_{Z} k(t, y) \eta(d t, d y):=\sum_{0 \leq t \leq T} k\left(t, \triangle Y_{t}\right) \chi_{Z}\left(\triangle Y_{t}\right)
$$

where

$$
Y_{t}:=\int_{Z} y \eta(t, d y)=\sum_{0 \leq t \leq t} \triangle L_{s} \chi_{Z}\left(\triangle L_{t}\right)
$$

as a random finite sum which enables us to define

$$
\left.\int_{0}^{T} \int_{Z} k(t, y) \widetilde{\eta}(d t, d y):=\int_{0}^{T} \int_{Z} k(t, y) \eta(d t, d y)-\int_{0}^{T} \int_{Z} k(t, y)\right) \lambda(d y) d t
$$

Furthermore, we can see that $\int_{0}^{t} \int_{Z} k(s, y) \widetilde{\eta}(d s, d y)$ is an $\mathbb{H}$-valued centered square-integrable martingale such that 


$$
\mathbf{E}\left(\left|\int_{0}^{T} \int_{Z} k(t, y) \widetilde{\eta}(d t, d y)\right|_{\mathbb{H}}^{2}\right)=\int_{0}^{T} \int_{Z} \mathbf{E}|k(t, y)|_{\mathbb{H}}^{2} \lambda(d y) d t
$$

We can refer to Protter [37] for a systematic theory about stochastic integrals of this kind.

Thus, the stochastic 2D Navier-Stokes equations with with memory and Poisson jumps (1) can be rewritten as follows in the abstract mathematical setting:

$$
\left\{\begin{aligned}
d X(t)= & {[-v A X(t)-B(X(t))+f(x)+g(X(t-\rho(t)))] d t+\sigma(t, X(t-\delta(t))) d W(t) } \\
& +\int_{Z} k(t, X(t-\gamma(t)), z) \widetilde{\eta}(d t, d z) \quad t \geq 0, \\
X(\theta)= & \phi(\theta) \in L^{2}(\Omega, \mathcal{C}([-r, 0], \mathbb{H})), \quad \theta \in[-r, 0], \quad r>0
\end{aligned}\right.
$$

where $f \in \mathbb{V}^{*}, g: \mathbb{V} \rightarrow \mathbb{V}^{*}, \sigma:[0,+\infty) \times \mathbb{V} \rightarrow \mathcal{L}_{2}^{0}(\mathbb{K}, \mathbb{H})$, and $k:[0,+\infty) \times \mathbb{H} \times(\mathbb{K}-\{0\}) \rightarrow$ $\mathbb{H}$ are progressively measurable; $L^{2}(\Omega, \mathcal{C}([-r, 0], \mathbb{H}))$ denotes the family of all almost surely bounded $\left(\mathcal{F}_{t}\right)_{t \geqslant 0}$-measurable and $\mathcal{C}([-r, 0], \mathbb{H})$-valued stochastic process, here $\mathcal{C}([-r, 0], \mathbb{H})$ denote the family of all right-continuous functions with left-hand limits $\phi$ from $[-r, 0]$ to $\mathbb{H}$ which is equipped with the norm $\|\phi\|_{\mathcal{C}}:=\sup _{-r \leq \theta \leq 0}|\phi(\theta)|$.

Now, we give the definition of the weak solution of system (2).

Definition 1. An $\mathcal{F}_{t}$-adapted process $X(t)$ is called the weak solution to (2) if the following conditions are satisfied:

(i) $X(t) \in \mathcal{C}(-r, T ; \mathbb{H}) \cap L^{2}(-r, T ; \mathbb{V})$, a.s., $\forall T>0$;

(ii) the following integral equation holds as an identity in $\mathbb{V}^{*}$ a.s., $\forall t \in[0, T]$,

$$
\begin{aligned}
X(t)=X(0) & +\int_{0}^{t}[-v A X(s)-B(X(s))+f(s)+g(X(s-\rho(s)))] d s \\
& +\int_{0}^{t} \sigma(s, X(s-\delta(s))) d W(s)+\int_{0}^{t} \int_{Z} k(s, X(s-\gamma(s)), z) \widetilde{\eta}(d s, d z) .
\end{aligned}
$$

For our purpose, we recall the Itô formula, which will play a key role in what follows. Let $C^{2}\left(\mathbb{H} ; \mathbb{R}^{+}\right)$ denote the space of all real-valued nonnegative functions $\mathrm{Y}$ on $\mathbb{H}$ with the following properties:

(a) $\mathrm{Y}(x)$ is twice (Fréchet) differentiable in $x$;

(b) Both $Y_{x}(x)$ and $Y_{x x}(x)$ are continuous in $\mathbb{H}$ and $\mathcal{L}(\mathbb{H})$.

Lemma 1. [40] Suppose $Y \in C^{2}\left(\mathbb{H} ; \mathbb{R}^{+}\right)$and $X(t), t \geq 0$, is a weak solution to (2). Then

$$
\begin{aligned}
Y(X(t))=\mathrm{Y} & (X(0))+\int_{0}^{t} \mathcal{L} \mathrm{Y}(\mathrm{X}(\mathrm{s})) d s+\int_{0}^{t}\left\langle\mathrm{Y}_{x}(\mathrm{X}(\mathrm{s})), \sigma(s, X(s-\delta(s))) d W(s)\right\rangle \\
& +\int_{0}^{t} \int_{\mathrm{Z}}[\mathrm{Y}(\mathrm{X}(\mathrm{s})+k(s, X(s-\gamma(s)), z))-\mathrm{Y}(\mathrm{X}(\mathrm{s}))] \widetilde{\eta}(d s, d z),
\end{aligned}
$$

where $\mathcal{L}$ is the associated diffusion operator defined, for any $x \in \mathbb{V}$, by

$$
\begin{aligned}
\mathcal{L} \mathrm{Y}(x(t))=\langle & \left.-v A x(t)-B(x(t))+f(t)+g(x(t-\rho(t))), \mathrm{Y}_{x}(x(t))\right\rangle \\
& +\frac{1}{2} \operatorname{trace}\left(\mathrm{Y}_{x x}(x(t)) \sigma(t, x(t-\delta(t))) Q \sigma(t, x(t-\delta(t)))^{*}\right) \\
& +\int_{\mathrm{Z}}\left[\mathrm{Y}(x(t)+k(s, x(s-\gamma(s)), z))-\mathrm{Y}(x(t))-\left\langle\mathrm{Y}_{x}(x(t)), k(s, x(s-\gamma(s)), z)\right\rangle\right] \lambda(d z) .
\end{aligned}
$$

Definition 2. We say that a weak solution $X(t)$ of system (2) converges to $u_{\infty} \in \mathbb{H}$ exponentially in the mean square if there exist $c>0$ and $M_{0}>0$ such that for all $t \geq 0$

$$
\mathbf{E}\left|X(t)-u_{\infty}\right|^{2} \leq M_{0} e^{-c t}
$$

In particular, if $u_{\infty}$ is a solution to (2), then it is said that $u_{\infty}$ is exponentially stable in the mean square provided that every weak solution to (2) converges to $u_{\infty}$ exponentially in the mean square with the same exponential order $c>0$. 
Definition 3. We say that a weak solution $X(t)$ of system (2) converges to $u_{\infty} \in \mathbb{H}$ almost surely exponentially if there exist $\gamma>0$ such that

$$
\lim _{t \rightarrow \infty} \frac{\log \left|X(t)-u_{\infty}\right|}{t} \leq-\gamma, \quad \text { a.s.. }
$$

In particular, if $u_{\infty}$ is a solution to (2), then it is said that $u_{\infty}$ is almost surely exponentially stable provided that every weak solution to (2) converges to $u_{\infty}$ almost surely exponentially with the same constant $\gamma$.

\section{Main results}

In this section, we will discuss the asymptotic behavior for the weak solutions of stochastic 2D Navier-Stokes equations with finite memory and Poisson jumps.

Let $\lambda_{1}>0$ be the first eigenvalue of $A$, then

$$
\|v\|^{2} \geq \lambda_{1}|v|^{2}, \quad \forall v \in V
$$

To investigate the asymptotic behavior for the weak solutions of (2), we assume the following hypotheses:

(H1) $g(0)=0$ and there exists a positive number $C_{g}$ such that

$$
\|g(u)-g(v)\|_{\mathbb{V}^{*}} \leq C_{g}|u-v|, \quad \forall u, v \in \mathbb{H} .
$$

(H2) There exist integrable functions $\alpha_{1}, \gamma_{1}:[0, \infty) \rightarrow \mathbb{R}^{+}$such that, for certain constant $\beta_{1} \geq 0$ and $u \in \mathbb{H}$,

$$
\|\sigma(t, u)\|_{\mathcal{L}_{2}^{0}}^{2} \leq \alpha_{1}(t)+\left(\beta_{1}+\gamma_{1}(t)\right)\left|u-u_{\infty}\right|^{2} .
$$

(H3) There exist integrable functions $\alpha_{2}, \gamma_{2}:[0, \infty) \rightarrow \mathbb{R}^{+}$such that, for certain constant $\beta_{2} \geq 0$ and $u \in \mathbb{H}$,

$$
\int_{Z}|k(t, u, z)|^{2} \lambda(d z) \leq \alpha_{2}(t)+\left(\beta_{2}+\gamma_{2}(t)\right)\left|u-u_{\infty}\right|^{2} .
$$

(H4) There exists $\theta>0$ such that, for $t \geq 0$,

$$
\int_{0}^{\infty} e^{\theta s} \alpha_{i}(t) d t<\infty, \quad \int_{0}^{\infty} e^{\theta s} \gamma_{i}(t) d t<\infty, \quad i=1,2 .
$$

We first consider the existence of the stationary solution to the equation

$$
v A X+B X=f(x)+g(X) \quad\left(\text { in } \mathbb{V}^{*}\right) .
$$

We have the following lemma:

Lemma 2. [20] Suppose that $g$ satisfies the condition (H1) and $v>\lambda_{1}^{-1} C_{g}$. Then we have the following:

(i) For all $f \in \mathbb{V}^{*}$, there exists a stationary solution $u_{\infty}$ to (4).

(ii) There exists a constant $C(D)>0$ such that, if $\left(v-\lambda_{1}^{-1} C_{g}\right)^{2}>C(D)\|f\|_{\mathbb{V}^{*}}$, then the stationary solution to (4) is unique.

Now, using above lemma, we will discuss the asymptotic behavior for the weak solutions of (2). Hence, throughout this paper we assume that there exists a unique stationary solution $u_{\infty} \in \mathbb{V}$ to (4).

Set $y(t):=X(t)-u_{\infty}$ and $Y(y(t))=y^{2}(t)$. Then the function $y(t)$ satisfies the following equation:

$$
\begin{aligned}
d\left(X(t)-u_{\infty}\right)= & {\left[-v A\left(X(t)-u_{\infty}\right)\right)-\left(B\left(X(t)-B\left(u_{\infty}\right)\right)+\left(g(X(t-\rho(t)))-g\left(u_{\infty}\right)\right)\right] d t } \\
& +\sigma(t, X(t-\delta(t))) d W(t)+\int_{Z} k(t, X(t-\gamma(t)), v) \widetilde{\eta}(d t, d v) .
\end{aligned}
$$

Similar to the articles of Caraballo et al., [7], Liu [41] and Wan [42], in which, they studied Gaussian white noise by Itô formula, utilizing Itô formula for Lévy noise in Lemma 1 to the function $Y(y(t))=y^{2}(t)$ and taking expectation, we easily obtain the following result. 
Theorem 1. Suppose that the conditions $(\mathbf{H 1})-(\mathbf{H} 4)$ hold. Then there exists a unique weak solution $X(t)-u_{\infty} \in$ $\mathcal{C}(-r, T ; \mathbb{H}) \cap L^{2}(-r, T ; \mathbb{V})$ a.s.. Furthermore, the following identity holds:

$$
\begin{aligned}
\frac{d}{d t} \mathbf{E}\left|X(t)-u_{\infty}\right|^{2}= & -2 \mathbf{E}\left\langle v A\left(X(t)-u_{\infty}\right), X(t)-u_{\infty}\right\rangle-2 \mathbf{E}\left\langle B\left(X(t)-B\left(u_{\infty}\right), X(t)-u_{\infty}\right\rangle\right. \\
& +2 \mathbf{E}\left\langle g(X(t-\rho(t)))-g\left(u_{\infty}\right), X(t)-u_{\infty}\right\rangle+\mathbf{E}\|\sigma(t, X(t-\delta(t)))\|_{\mathcal{L}_{2}^{0}}^{2} \\
& +\mathbf{E} \int_{Z}|k(t, X(t-\gamma(t)), z)|^{2} \lambda(d z) .
\end{aligned}
$$

The first main result of this section is the following theorem.

Theorem 2. Suppose that the conditions $(\mathbf{H 1})-(\mathbf{H} 4)$ hold. Then the weak solution $X(t)$ to (2) converges to the stationary solution $u_{\infty}$ to (4) exponentially stable in the mean square provided that the following inequality

$$
C(D) \sqrt{\lambda_{1}}\left\|u_{\infty}\right\|+C_{g}+\frac{\beta_{1}+\beta_{2}}{2}<v \lambda_{1}
$$

holds.

Proof. From (5), there exists a positive constant $v$ such that

$$
C(D) \sqrt{\lambda_{1}}\left\|u_{\infty}\right\|+C_{g}+\frac{\beta_{1}+\beta_{2}}{2}<C(D) \sqrt{\lambda_{1}}\left\|u_{\infty}\right\|+\frac{\beta_{1}+\beta_{2}}{2}+\frac{1}{2 v}+\frac{v C_{g}^{2}}{2}<v \lambda_{1} .
$$

Furthermore, there exists a constant $c \in(0, \theta)$ sufficiently small such that

$$
c+2 C(D) \sqrt{\lambda_{1}}\left\|u_{\infty}\right\|+\beta_{1}+\beta_{2}+\frac{1}{2 v}+v C_{g}^{2} e^{c r}-2 v \lambda_{1}<0 .
$$

For convenience, we shall denote

$$
\alpha(t)=\alpha_{1}(t)+\alpha_{2}(t), \quad \eta(t)=\left(\gamma_{1}(t)+\gamma_{2}(t)\right) e^{c r} .
$$

Since $\alpha_{i}, \gamma_{i}, i=1,2$, is integrable, together with the assumption (H4), we deduce that

$$
\Lambda_{1}=\int_{0}^{\infty} \eta(t) d t<\infty, \quad \Lambda_{2}=\int_{0}^{\infty} \alpha(t) d t \leq \Lambda_{3}=\int_{0}^{\infty} \alpha(t) e^{\theta t} d t<\infty .
$$

Define the function

$$
F(t):=\left\{\begin{array}{l}
\mathbf{E}\left|X(t)-u_{\infty}\right|^{2} e^{c t} \exp \left(-\int_{0}^{t}\left[\eta(s)+\alpha(s) e^{c s}\right] d s\right), \quad t \geq 0, \\
\mathbf{E}\left|X(t)-u_{\infty}\right|^{2} e^{c t}, \quad t \in[-r, 0) .
\end{array}\right.
$$

Clearly, $F(t)$ is right-continuous functions with left-hand limits on $[-r,+\infty)$ and

$$
\frac{d F(t)}{d t}=e^{c t} \exp \left(-\int_{0}^{t}\left[\eta(s)+\alpha(s) e^{c s}\right] d s\right)\left\{\left[c-\eta(t)-\alpha(t) e^{c t}\right] \mathbf{E}\left|X(t)-u_{\infty}\right|^{2}+\frac{d}{d t} \mathbf{E}\left|X(t)-u_{\infty}\right|^{2}\right\}
$$

Now, from definition on the operator $B$, we get

$$
\left\langle B(X(s))-B\left(u_{\infty}\right), X(s)-u_{\infty}\right\rangle=b\left(X(s)-u_{\infty}, u_{\infty}, X(s)-u_{\infty}\right),
$$

and from the properties on trilinear form $b$, we obtain

$$
\begin{aligned}
\left|b\left(X(s)-u_{\infty}, u_{\infty}, X(s)-u_{\infty}\right)\right| & \leq C_{1}\left|X(s)-u_{\infty}\right|^{\frac{1}{2}}\left\|X(s)-u_{\infty}\right\|^{\frac{1}{2}}\left\|u_{\infty}\right\|\left|X(s)-u_{\infty}\right|^{\frac{1}{2}}\left\|X(s)-u_{\infty}\right\|^{\frac{1}{2}} \\
& =C_{1}\left|X(s)-u_{\infty}\right|\left\|X(s)-u_{\infty}\right\|\left\|u_{\infty}\right\| \\
& \leq C_{1} \lambda_{1}^{-\frac{1}{2}}\left\|u_{\infty}\right\|\left\|X(s)-u_{\infty}\right\|^{2} .
\end{aligned}
$$


Furthermore, by Young's inequality and the assumption (H1), we have

$$
\left|\left\langle g(X(s-\rho(s)))-g\left(u_{\infty}\right), X(s)-u_{\infty}\right\rangle\right| \leq v C_{g}^{2}\left|X(s-\rho(s))-u_{\infty}\right|^{2}+\frac{1}{v}\left|X(s)-u_{\infty}\right|^{2} .
$$

Hence,

$$
\begin{aligned}
\frac{d F(t)}{d t} \leq & e^{c t} \exp \left(-\int_{0}^{t}\left[\eta(s)+\alpha(s) e^{c s}\right] d s\right)\left\{\left[c-\eta(t)-\alpha(t) e^{c t}-2 v \lambda_{1}\right.\right. \\
& \left.+2 C(D) \sqrt{\lambda_{1}}\left\|u_{\infty}\right\|+\frac{1}{v}\right] \mathbf{E}\left|X(t)-u_{\infty}\right|^{2}+v C_{g}^{2} \mathbf{E}\left|X(t-\rho(t))-u_{\infty}\right|^{2} \\
& \left.+\mathbf{E}\|\sigma(t, X(t-\delta(t)))\|_{\mathcal{L}_{2}^{0}}^{2}+\mathbf{E} \int_{Z}|k(t, X(t-\gamma(t)), z)|^{2} \lambda(d z)\right\} \\
\leq & \left(c+2 C(D) \sqrt{\lambda_{1}}\left\|u_{\infty}\right\|+\frac{1}{v}-\eta(t)-2 v \lambda_{1}\right) F(t)+\alpha(t) e^{c t}-\alpha(t) e^{c t} F(t) \\
& +e^{c t} \exp \left(-\int_{0}^{t}\left[\eta(s)+\alpha(s) e^{c s}\right] d s\right) v C_{g}^{2} \mathbf{E}\left|X(t-\rho(t))-u_{\infty}\right|^{2} \\
& +e^{c t} \exp \left(-\int_{0}^{t}\left[\eta(s)+\alpha(s) e^{c s}\right] d s\right)\left[\beta_{1}+\gamma_{1}(t)\right] \mathbf{E}\left|X(t-\delta(t))-u_{\infty}\right|^{2} \\
& +e^{c t} \exp \left(-\int_{0}^{t}\left[\eta(s)+\alpha(s) e^{c s}\right] d s\right)\left[\beta_{2}+\gamma_{2}(t)\right] \mathbf{E}\left|X(t-\gamma(t))-u_{\infty}\right|^{2} .
\end{aligned}
$$

In what follows, we claim that for any $t \geq 0$

$$
F(t) \leq \widetilde{M}:=1+\sup _{[-r, 0]} \mathbf{E}\left|X(t)-u_{\infty}\right|^{2} .
$$

If the inequality (12) does not hold, then there exists $t^{*}>0$ such that, for any $\varepsilon>0$

$$
F(t)<\widetilde{M}, \quad 0 \leq t<t^{*}, \quad F\left(t^{*}\right)=\widetilde{M}, \quad F(t)>\widetilde{M}, \quad t^{*} \leq t \leq t^{*}+\varepsilon .
$$

This, in addition to (8), it can be shown that

$$
\frac{d F\left(t^{*}\right)}{d t} \geq 0
$$

Furthermore,

$$
\begin{aligned}
\frac{d F\left(t^{*}\right)}{d t} \leq & \left(c+2 C(D) \sqrt{\lambda_{1}}\left\|u_{\infty}\right\|+\frac{1}{v}-\eta\left(t^{*}\right)-2 v \lambda_{1}\right) F\left(t^{*}\right)+\alpha\left(t^{*}\right) e^{c t^{*}}-\alpha\left(t^{*}\right) e^{c t^{*}} F\left(t^{*}\right) \\
& +e^{c t^{*}} \exp \left(-\int_{0}^{t^{*}}\left[\eta(s)+\alpha(s) e^{c s}\right] d s\right) v C_{g}^{2} \mathbf{E}\left|X\left(t^{*}-\rho\left(t^{*}\right)\right)-u_{\infty}\right|^{2} \\
& +e^{c t^{*}} \exp \left(-\int_{0}^{t^{*}}\left[\eta(s)+\alpha(s) e^{c s}\right] d s\right)\left[\beta_{1}+\gamma_{1}\left(t^{*}\right)\right] \mathbf{E}\left|X\left(t^{*}-\delta\left(t^{*}\right)\right)-u_{\infty}\right|^{2} \\
& +e^{c t^{*}} \exp \left(-\int_{0}^{t^{*}}\left[\eta(s)+\alpha(s) e^{c s}\right] d s\right)\left[\beta_{2}+\gamma_{2}\left(t^{*}\right)\right] \mathbf{E}\left|X\left(t^{*}-\gamma\left(t^{*}\right)\right)-u_{\infty}\right|^{2} .
\end{aligned}
$$

Next, we split the following cases to derive the desired assertion.

Case 1: If $t^{*}-\rho\left(t^{*}\right) \geq 0, t^{*}-\delta\left(t^{*}\right) \geq 0, t^{*}-\gamma\left(t^{*}\right) \geq 0$, we then have from (6) and (13) that

$$
\begin{aligned}
\frac{d F\left(t^{*}\right)}{d t} \leq & \left(c+2 C(D) \sqrt{\lambda_{1}}\left\|u_{\infty}\right\|+\frac{1}{v}-\eta\left(t^{*}\right)-2 v \lambda_{1}\right) F\left(t^{*}\right)+\alpha\left(t^{*}\right) e^{c t^{*}}-\alpha\left(t^{*}\right) e^{c t^{*}} F\left(t^{*}\right) \\
& +e^{c \rho\left(t^{*}\right)} \exp \left(-\int_{t^{*}-\rho\left(t^{*}\right)}^{t^{*}}\left[\eta(s)+\alpha(s) e^{c s}\right] d s\right) v C_{g}^{2} F\left(X\left(t^{*}-\rho\left(t^{*}\right)\right)\right) \\
& +e^{c \delta\left(t^{*}\right)} \exp \left(-\int_{t^{*}-\delta\left(t^{*}\right)}^{t^{*}}\left[\eta(s)+\alpha(s) e^{c s}\right] d s\right)\left[\beta_{1}+\gamma_{1}\left(t^{*}\right)\right] F\left(X\left(t^{*}-\delta\left(t^{*}\right)\right)\right) \\
& +e^{c \gamma\left(t^{*}\right)} \exp \left(-\int_{t^{*}-\gamma\left(t^{*}\right)}^{t^{*}}\left[\eta(s)+\alpha(s) e^{c s}\right] d s\right)\left[\beta_{2}+\gamma_{2}\left(t^{*}\right)\right] F\left(X\left(t^{*}-\gamma\left(t^{*}\right)\right)\right)
\end{aligned}
$$




$$
\leq\left(c+2 C(D) \sqrt{\lambda_{1}}\left\|u_{\infty}\right\|+\beta_{1}+\beta_{2}+\frac{1}{v}+v C_{g}^{2} e^{c r}-2 v \lambda_{1}\right) \widetilde{M}<0,
$$

which contradicts with (14). That is, the desired assertion (12) must hold.

Case 2: If $t^{*}-\rho\left(t^{*}\right) \leq 0, t^{*}-\delta\left(t^{*}\right) \leq 0, t^{*}-\gamma\left(t^{*}\right) \leq 0$, we then have from (6) and (13) that

$$
\begin{aligned}
\frac{d F\left(t^{*}\right)}{d t} \leq & \left(c+2 C(D) \sqrt{\lambda_{1}}\left\|u_{\infty}\right\|+\frac{1}{v}-\eta\left(t^{*}\right)-2 v \lambda_{1}\right) F\left(t^{*}\right)+\alpha\left(t^{*}\right) e^{c t^{*}}-\alpha\left(t^{*}\right) e^{c t^{*}} F\left(t^{*}\right) \\
& +e^{c \rho\left(t^{*}\right)} \exp \left(-\int_{0}^{t^{*}}\left[\eta(s)+\alpha(s) e^{c s}\right] d s\right) v C_{g}^{2} F\left(X\left(t^{*}-\rho\left(t^{*}\right)\right)\right) \\
& +e^{c \delta\left(t^{*}\right)} \exp \left(-\int_{0}^{t^{*}}\left[\eta(s)+\alpha(s) e^{c s}\right] d s\right)\left[\beta_{1}+\gamma_{1}\left(t^{*}\right)\right] F\left(X\left(t^{*}-\delta\left(t^{*}\right)\right)\right) \\
& +e^{c \gamma\left(t^{*}\right)} \exp \left(-\int_{0}^{t^{*}}\left[\eta(s)+\alpha(s) e^{c s}\right] d s\right)\left[\beta_{2}+\gamma_{2}\left(t^{*}\right)\right] F\left(X\left(t^{*}-\gamma\left(t^{*}\right)\right)\right) \\
\leq & \left(c+2 C(D) \sqrt{\lambda_{1}}\left\|u_{\infty}\right\|+\beta_{1}+\beta_{2}+\frac{1}{v}+v C_{g}^{2} e^{c r}-2 v \lambda_{1}\right) \widetilde{M}<0 .
\end{aligned}
$$

This is a contradiction. Hence, (12) holds true for any $t \geq 0$. For other cases, for example: $t^{*}-\rho\left(t^{*}\right) \geq$ $0, t^{*}-\delta\left(t^{*}\right) \leq 0, t^{*}-\gamma\left(t^{*}\right) \leq 0, \ldots$, in the same way as Cases 1 and 2 were done, we can show (12).

Therefore, from (12), we infer that

$$
\mathbf{E}\left|X(t)-u_{\infty}\right|^{2} \leq \widetilde{M} e^{-c t} \exp \left(\int_{0}^{t}\left[\eta(s)+\alpha(s) e^{c s}\right] d s\right) \stackrel{(3.4)}{\leq} \widetilde{M} e^{\Lambda_{1}+\Lambda_{3}} e^{-c t}, \quad \forall t \geq 0 .
$$

This completes the proof of the theorem.

In the following, $C$ will denote a generic constant whose values might change from line to line.

In order to prove the almost surely exponential stability of the weak solution of (2) we shall establish the following lemma.

Lemma 3. For any $t \geq 0$, there exists a constant $C>0$ such that

$$
\begin{aligned}
& \mathbf{E} \sup _{\tau \in[0, t]}\left|\int_{0}^{\tau} \int_{Z}\left(|k(s, X(s-\gamma(s)), z)|_{\mathbb{H}}^{2}+2\langle X(s-), k(s, X(s-\gamma(s)), z)\rangle_{\mathbb{H}}\right) \widetilde{\eta}(d s, d z)\right| \\
& \leq C \mathbf{E} \int_{0}^{t} \int_{Z}|k(\tau, X(\tau-\gamma(\tau)), z)|_{\mathbb{H}}^{2} \lambda(d z) d \tau+\frac{1}{4} \mathbf{E} \sup _{\tau \in[0, t]}|X(\tau)|_{\mathbb{H}}^{2} .
\end{aligned}
$$

Proof. Set

$$
J_{t}:=\int_{0}^{\tau} \int_{Z}\left(\|\gamma(s, X(s-\rho(s)), z)\|_{\mathbb{H}}^{2}+2\langle X(s-), \gamma(s, X(s-\rho(s)), z)\rangle_{\mathbb{H}}\right) \widetilde{\eta}(d s, d z)
$$

Then,

$$
\begin{aligned}
{[J, J]_{t}^{\frac{1}{2}} } & =\left\{\sum_{s \in D_{p, s \leq t}}\left(|\gamma(s, X(s-\rho(s)), p(s))|_{\mathbb{H}}^{2}+2\langle X(s-), \gamma(s, X(s-\rho(s)), p(s))\rangle_{\mathbb{H}}\right)^{2}\right\}^{\frac{1}{2}} \\
& \leq C\left(\sum_{s \in D_{p, s} \leq t}|\gamma(s, X(s-\rho(s)), p(s))|_{\mathbb{H}}^{4}\right)^{\frac{1}{2}}+C\left(\sum_{s \in D_{p, s \leq t}}|X(s-)|_{\mathbb{H}}^{2}|\gamma(s, X(s-\rho(s)), p(s))|_{\mathbb{H}}^{2}\right)^{\frac{1}{2}} \\
& \leq C \sum_{s \in D_{p, s \leq t}}|\gamma(s, X(s-\rho(s)), p(s))|_{\mathbb{H}}^{2}+C \sup _{0 \leq s \leq t}\left(|X(s-)|_{\mathbb{H}}\right)\left(\sum_{s \in D_{p}, s \leq t}|\gamma(s, X(s-\rho(s)), p(s))|_{\mathbb{H}}^{2}\right)^{\frac{1}{2}} \\
& \leq C \sum_{s \in D_{p, s \leq t}}|\gamma(s, X(s-\rho(s)), p(s))|_{\mathbb{H}}^{2}+\frac{1}{4} \sup _{0 \leq s \leq t}\left(|X(s-)|_{\mathbb{H}}^{2}\right) .
\end{aligned}
$$

By Burkholder-Davis-Gundy inequality [43], we obtain that 


$$
\begin{aligned}
\left.\operatorname{E}_{\tau \in[0, t]} \sup _{\tau}\left|J_{\tau}\right|\right] & \leq \mathrm{CE}\left([J, J]_{t}^{\frac{1}{2}}\right) \\
& \leq \mathrm{CE}\left(\sum_{s \in D_{p, s} \leq t}|\gamma(s, X(s-\rho(s)), p(s))|_{\mathbb{H}}^{2}\right)+\frac{1}{4} \mathbf{E}\left(\sup _{0 \leq s \leq t}\left(|X(s-)|_{\mathbb{H}}^{2}\right)\right) \\
& =C \mathbf{E} \int_{0}^{t} \int_{Z}|k(\tau, X(\tau-\gamma(\tau)), z)|_{\mathbb{H}}^{2} \lambda(d z) d \tau+\frac{1}{4} \mathbf{E} \sup _{\tau \in[0, t]}|X(\tau)|_{\mathbb{H}}^{2} .
\end{aligned}
$$

The proof is therefore complete.

We have the following theorem:

Theorem 3. Assume that all the assumptions of Theorem 2 are satisfied. Then, any weak solution $X(t)$ to (2) converges to the stationary solution $u_{\infty}$ of (4) almost surely exponentially.

Proof. Let $n_{1}, n_{2}$ and $n_{3}$ be positive integers such that

$$
n_{1}-\rho\left(n_{1}\right) \geq n_{1}-r \geq 1, \quad n_{2}-\delta\left(n_{2}\right) \geq n_{2}-r \geq 1, \quad n_{3}-\gamma\left(n_{3}\right) \geq n_{3}-r \geq 1 .
$$

Set $n=\max \left\{n_{1}, n_{2}, n_{3}\right\}$. By the Itô formula, it follows for any $t \geq n$,

$$
\begin{aligned}
\left|X(t)-u_{\infty}\right|^{2}= & \left|X(n)-u_{\infty}\right|^{2}-2 \int_{n}^{t}\left\langle v A\left(X(s)-u_{\infty}\right), X(s)-u_{\infty}\right\rangle d s-2 \int_{n}^{t}\left\langle B(X(s))-B\left(u_{\infty}\right), X(s)-u_{\infty}\right\rangle d s \\
& +2 \int_{n}^{t}\left\langle g(X(s-\rho(s)))-g\left(u_{\infty}\right), X(s)-u_{\infty}\right\rangle d s+\int_{n}^{t}\|\sigma(s, X(s-\delta(s)))\|_{\mathcal{L}_{2}^{0}}^{2} d s \\
& +2 \int_{n}^{t}\left\langle X(s)-u_{\infty}, \sigma(s, X(s-\delta(s))) d W(s)\right\rangle+\int_{n}^{t} \int_{Z}|k(s, X(s-\gamma(s)), z)|^{2} \lambda(d z) d s \\
& +\int_{n}^{t} \int_{Z}\left[|k(s, X(s-\gamma(s)), z)|^{2}+2 \int_{n}^{t}\left\langle X(s)-u_{\infty}, k(s, X(s-\gamma(s)), z)\right\rangle\right] \widetilde{\eta}(d s, d z) .
\end{aligned}
$$

In view of Burkholder-Davis-Gundy inequality and the Young inequality, we have

$$
\begin{aligned}
& 2 \mathbf{E}\left[\sup _{t \in[n, n+1]} \int_{n}^{t}\left\langle X(s)-u_{\infty}, \sigma(s, X(s-\rho(s))) d W(s)\right\rangle\right] \\
& \leq 8 \mathbf{E}\left[\int_{n}^{n+1}\left|X(s)-u_{\infty}\right|^{2}\|\sigma(s, X(s-\rho(s)))\|_{\mathcal{L}_{2}^{0}}^{2} d s\right]^{\frac{1}{2}} \\
& \leq \frac{1}{2} \mathbf{E}\left(\sup _{t \in[n, n+1]}\left|X(t)-u_{\infty}\right|^{2}\right)+32 \int_{n}^{n+1} \mathbf{E}\|\sigma(s, X(s-\rho(s)))\|_{\mathcal{L}_{2}^{0}}^{2} d s .
\end{aligned}
$$

Applying Lemma 2, for any $t \geq 0$ we can get

$$
\begin{aligned}
& \mathbf{E} \sup _{t \in[n, n+1]} \int_{n}^{t} \int_{Z}\left(|k(s, X(s-\gamma(s)), z)|^{2}+2\left\langle X\left(s-u_{\infty}\right), k(s, X(s-\gamma(s)), z)\right\rangle\right) \widetilde{\eta}(d s, d z) \\
& \leq C \mathbf{E} \int_{n}^{n+1} \int_{Z}|k(s, X(s-\gamma(s)), z)|^{2} \lambda(d z) d s+\frac{1}{4} \mathbf{E}\left(\sup _{t \in[n, n+1]}\left|X(t)-u_{\infty}\right|^{2}\right) .
\end{aligned}
$$

Hence,

$$
\begin{aligned}
\mathbf{E}\left(\sup _{t \in[n, n+1]}\left|X(t)-u_{\infty}\right|^{2}\right) \leq & \mathbf{E}\left|X(n)-u_{\infty}\right|^{2}+\left(2 C(D) \sqrt{\lambda_{1}}\left\|u_{\infty}\right\|+\frac{1}{v}-2 v \lambda_{1}\right) \int_{n}^{n+1} \mathbf{E}\left|X(s)-u_{\infty}\right|^{2} d s \\
& +v C_{g}^{2} \int_{n}^{n+1} \mathbf{E}\left|X(s-\rho(s))-u_{\infty}\right|^{2} d s+33 \int_{n}^{n+1} \mathbf{E}\|\sigma(s, X(s-\delta(s)))\|_{\mathcal{L}_{2}^{0}}^{2} d s \\
& +(C+1) \mathbf{E} \int_{n}^{n+1} \int_{Z}|k(s, X(s-\gamma(s)), z)|^{2} \lambda(d z) d s+\frac{3}{4} \mathbf{E}\left(\sup _{t \in[n, n+1]}\left|X(t)-u_{\infty}\right|^{2}\right) .
\end{aligned}
$$


This implies that

$$
\begin{aligned}
\mathbf{E}\left(\sup _{t \in[n, n+1]}\left|X(t)-u_{\infty}\right|^{2}\right) \leq & 4 \mathbf{E}\left|X(n)-u_{\infty}\right|^{2}+4\left(2 C(D) \sqrt{\lambda_{1}}\left\|u_{\infty}\right\|+\frac{1}{v}-2 v \lambda_{1}\right) \int_{n}^{n+1} \mathbf{E}\left|X(s)-u_{\infty}\right|^{2} d s \\
& +4 v C_{g}^{2} \int_{n}^{n+1} \mathbf{E}\left|X(s-\rho(s))-u_{\infty}\right|^{2} d s \\
& +132 \int_{n}^{n+1}\left[\alpha_{1}(s)+\left(\beta_{1}+\gamma_{1}(s)\right) \mathbf{E}\left|X(s-\delta(s))-u_{\infty}\right|^{2}\right] d s \\
& +4(C+1) \int_{n}^{n+1}\left[\alpha_{2}(s)+\left(\beta_{2}+\gamma_{2}(s)\right) \mathbf{E}\left|X(s-\gamma(s))-u_{\infty}\right|^{2}\right] d s
\end{aligned}
$$

On the other hand, from Theorem 2, it is easy to show that

$$
\mathbf{E}\left(\sup _{t \in[n, n+1]}\left|X(t)-u_{\infty}\right|^{2}\right) \leq 4 M_{0} e^{-c n}+4 \int_{n}^{n+1} M_{0} e^{-c s}\left[\gamma^{*}(s)+\alpha^{*}(s) e^{c s}\right] d s,
$$

where

$$
\begin{gathered}
\alpha^{*}(t):=33 \alpha_{1}(t)+4(C+1) \alpha_{2}(t) \\
\gamma^{*}(t):=2 C(D) \sqrt{\lambda_{1}}\left\|u_{\infty}\right\|+\frac{1}{v}-2 v \lambda_{1}+\left[v C_{g}^{2}+33\left(\beta_{1}+\gamma_{1}(t)\right)+(C+1)\left(\beta_{2}+\gamma_{2}(t)\right)\right] e^{c r} .
\end{gathered}
$$

In view of assumption (H4), there exists a positive constant $\Lambda$ such that we obtain that

$$
\mathbf{E}\left(\sup _{t \in[n, n+1]}\left|X(t)-u_{\infty}\right|^{2}\right) \leq 4 M_{0} e^{-c n}\left(1+\frac{\Lambda}{c}\right) .
$$

Let $\epsilon_{n}>0$ be any fixed positive real number. Then by Chebychev's inequality, we deduce that

$$
\mathbf{P}\left\{\sup _{t \in[n, n+1]}\left|X(t)-u_{\infty}\right|>\epsilon_{n}\right\} \leq \frac{4 M_{0} e^{-c n}\left(1+\frac{\Lambda}{c}\right)}{\epsilon_{n}^{2}} .
$$

Therefore, since $\epsilon_{n}$ is any fixed real number, let $\epsilon_{n}=e^{-\frac{(c-\varepsilon) n}{4}}$, where $\varepsilon \in(0, c)$. Then by the Borel-Cantelli lemma [44], we can yield that

$$
\varlimsup_{t \rightarrow \infty} \frac{\log \left|X(t)-u_{\infty}\right|}{t} \leq-\frac{c-\varepsilon}{4}, \text { a.s.. }
$$

Letting $\varepsilon \rightarrow 0^{+}$, this completes the proof of the theorem.

Remark 1. We consider a special case of the system (1) with variable delays when $\rho \equiv \delta$ and $\gamma \equiv 0$. That is, our system (1) reduces to the following system

$$
\left\{\begin{array}{l}
d X=[v \triangle X-\langle X, \nabla\rangle X-\nabla p+f(t)+g(X(t-\rho(t)))] d t+\sigma(X(t-\rho(t))) d W(t) \\
\operatorname{div} X=0 \text { in }(0,+\infty) \times D, \quad X(t, x)=0 \quad \text { on } \quad(0,+\infty) \times \partial D \\
X(0, x)=X_{0}(x), \quad X(t, x)=\phi(t, x), \quad(t, x) \in(-r, 0) \times D
\end{array}\right.
$$

The system (16) has been recently studied by Chen [22]. Furthermore, Wan and Zhou [23] discussed the following system

$$
\left\{\begin{array}{l}
d X(t)=[-v A X(t)-B(X(t))+f(X(t))+g(X(\rho(t)))] d t+\sigma(t, X(t)) d W(t) \\
\operatorname{div} X=0 \text { in }(0,+\infty) \times D, \quad X(t, x)=0 \quad \text { on } \quad(0,+\infty) \times \partial D \\
X(t, x)=\phi(t, x), \text { for } x \in D, \text { and } t \in[-r, 0], \text { with } r>0
\end{array}\right.
$$


and Caraballo et al., [7] also studied the stability of the stationary solutions of the following stochastic 2D Navier-Stokes without memory:

$$
\left\{\begin{array}{l}
d X(t)=[-v A X(t)-B(X(t))+g(X(t))] d t+\sigma(t, X(t)) d W(t) \\
\operatorname{div} X=0 \text { in }(0,+\infty) \times D, \quad X(t, x)=0 \quad \text { on } \quad(0,+\infty) \times \partial D \\
X(0, x)=X_{0}(x), \quad x \in D
\end{array}\right.
$$

By using method in our paper, the conclusions of some theorems in the works $[7,22,23]$ also easily obtained. Obviously, our work have extended the asymptotic behavior results of above works to cover a class of much more general stochastic 2D Navier-Stokes equations with memory and discontinuous multiplicative noise.

Remark 2. If $\sigma \equiv 0$ in the system (16), then by utilizing the direct method, in [20], Caraballo and Real have considered the asymptotic behavior for the weak solutions and Taniguchi [35] have investigated exponential stability of energy solutions to 2D stochastic functional Navier-Stokes equation perturbed by the Lévy process. However, unlike the works $[20,35]$, we need not require the function $\rho(t)$ to be differentiable and satisfies $0 \leq \rho^{\prime}(t)<1$. Therefore, our results extend and improve the one of Caraballo and Real [20] and Taniguchi [35].

Conflicts of Interest: "The author declares no conflict of interest."

\section{References}

[1] Lions, J. L. (1969). Quelques méthodes de résolution des problemes aux limites non linéaires. Springer.

[2] Temam, R. (1979). Navier-Stokes equations, Theory and Numerical Analysis, Second edition. North-Holland, Amsterdam.

[3] Rosa, R. (1998). The global attractor for the 2D Navier-Stokes flow on some unbounded domains. Nonlinear Analysis, 32(1), 71-86.

[4] Leray, J. (1933). Etude de diverses équations intégrales non linéaires et de quelques problèmes que pose l'hydrodynamique. Journal de Mathématiques Pures et Appliquées., 12, 1-82.

[5] Landau, L. D., \& Lifshitz, E. M. (1959). Fluid Mechanics, Vol. 6 of Course of Theoretical Physics, Pergamon, Oxford.

[6] Bensoussan, A., \& Temam, R. (1973). Equations stochastiques du type Navier-Stokes. Journal of Functional Analysis, 13(2), 195-222.

[7] Caraballo, T., Langa, J. A., \& Taniguchi, T. (2002). The exponential behaviour and stabilizability of stochastic 2D-Navier-Stokes equations. Journal of Differential Equations, 179(2), 714-737.

[8] Da Prato, G., \& Zabczyk, J. (1992). Stochastic Equations in Infinite Dimensions. Cambridge University Press, UK.

[9] Flandoli, F., \& Gatarek, D. (1995). Martingale and stationary solutions for stochastic Navier-Stokes equations. Probability Theory and Related Fields, 102(3), 367-391.

[10] Taniguchi, T. (2011). The existence of energy solutions to 2-dimensional non-Lipschitz stochastic Navier-Stokes equations in unbounded domains. Journal of Differential Equations, 251(12), 3329-3362.

[11] Caraballo, T., \& Shaikhet, L. (2014). Stability of delay evolution equations with stochastic perturbations. Communications on Pure and Applied Analysis, 13(5), 2095-2113.

[12] Guzzo, S. M., \& Planas, G. (2015). Existence of solutions for a class of Navier-Stokes equations with infinite delay. Advanced Nonlinear Studies, 94(4), 8401-855.

[13] Hale, J. K., Lunel, S. M. V., Verduyn, L. S., \& Lunel, S. M. V. (1993). Introduction to functional differential equations (Vol. 99). Springer Science \& Business Media.

[14] Huan, D. D. (2015). On the controllability of nonlocal second-order impulsive neutral stochastic integro-differential equations with infinite delay. Asian Journal of Control, 17(4), 1-10.

[15] Huan, D. D., \& Gao, H. (2015). A note on the existence of stochastic integro-differential equations with memory. Mathematical Methods in the Applied Sciences, 38(11), 2105-2119.

[16] Mao, X. (1997). Stochastic Differential Equations and Applications.

[17] Caraballo, T., \& Real, J. (2004). Attractors for 2D-Navier-Stokes models with delays. Journal of Differential Equations, 205(2), 271-297.

[18] García-Luengo, J., Marín-Rubio, P., \& Real, J. (2013). Pullback attractors for 2D Navier-Stokes equations with delays and their regularity. Advanced Nonlinear Studies, 13(2), 331-357.

[19] Marín-Rubio, P., \& Real, J. (2007). Attractors for 2D-Navier-Stokes equations with delays on some unbounded domains. Nonlinear Analysis, 67(10), 2784-2799. 
[20] Caraballo, T., \& Real, J. (2003). Asymptotic behaviour of two-dimensional Navier-Stokes equations with delays. Proceedings of the Royal Society of London. Series A: Mathematical, Physical and Engineering Sciences, 459(2040), 3181-3194.

[21] Planas, G., \& Hernández, E. (2008). Asymptotic behaviour of two-dimensional time-delayed Navier-Stokes equations. Discrete E Continuous Dynamical Systems, 21(4), 1245-1258.

[22] Chen, H. (2012). Asymptotic behavior of stochastic two-dimensional Navier-Stokes equations with delays. Proceedings-Mathematical Sciences, 122(2), 283-295.

[23] Wan, L., \& Zhou, Q. (2011). Asymptotic behaviors of stochastic two-dimensional Navier-Stokes equations with finite memory. Journal of Mathematical Physics, 52(4), 042703.

[24] Liu, L. F.,\& Caraballo, T. (2018). Analysis of a Stochastic 2D-Navier-Stokes Model with Infinite Delay. Journal of Dynamics and Differential Equations, 2018, 1-26.

[25] Razafimandimby, P. A., \& Sango, M. (2010). Asymptotic behavior of solutions of stochastic evolution equations for second grade fluids. Comptes Rendus Mathematique, 348(13-14), 787-790.

[26] Razafimandimby, P. A., \& Sango, M. (2012). On the exponential behaviour of stochastic evolution equations for non-Newtonian fluids. Applicable Analysis, 91(12), 2217-2233.

[27] Bessaih, H., Hausenblas, E., \& Razafimandimby, P. A. (2015). Strong solutions to stochastic hydrodynamical systems with multiplicative noise of jump type. Nonlinear Differential Equations and Applications NoDEA, 22(6), 1661-1697.

[28] Huan, D., \& Agarwal, A. (2014). Global attracting and quasi-invariant sets for stochastic Volterra-Levin equations with jumps. Dynamics of Continuous, Discrete and Impulsive Systems, Series A: Mathematical Analysis, 21, 343-353.

[29] Huan, D. D., \& Agarwal, R. P. (2015). Neutral SFDEs with jumps under Caratheodory conditions. Dynamics of Continuous, Discrete and Impulsive Systems, Series A: Mathematical Analysis, 22, 81-93.

[30] Huan, D. D., \& Agarwal, R. P. (2018). Asymptotic behavior, attracting and quasi-invariant sets for impulsive neutral SPFDE driven by Lévy noise. Stochastics and Dynamics, 18 (01), 1-21.

[31] Huan, D. D., \& Gao, H. (2015). Controllability of nonlocal second-order impulsive neutral stochastic functional integro-differential equations with delay and Poisson jumps. Cogent Engineering, 2(1), 1065585.

[32] Motyl, E.(2013). Stochastic Navier-Stokes equations driven by Lévy noise in unbounded 3D domains. Potential Analysis, 38(3), 863-912.

[33] Dong, Z., Li, W. V., \& Zhai, J. (2012). Stationary weak solutions for stochastic 3D Navier-Stokes equations with Lévy noise. Stochastics and Dynamics, 12(01), 1150006.

[34] Brzezniak, Z., Hausenblas, E., \& Zhu, J. (2013). 2D stochastic Navier-Stokes equations driven by jump noise. Nonlinear Analysis: Theory, Methods \& Applications, 79, 122-139.

[35] Taniguchi, T. (2012). The existence and asymptotic behaviour of energy solutions to stochastic 2D functional Navier-Stokes equations driven by Lévy processes. Journal of Mathematical Analysis and Applications, 385(2), 634-654.

[36] Peszat, S., \& Zabczyk, J. (2007). Stochastic partial differential equations with Lévy noise: An evolution equation approach (Vol. 113). Cambridge University Press.

[37] Protter, P. E. (2004). Stochastic integration and differential equations, Second edition. Springer, New York.

[38] Temam, R. (1988). Infinite Dimensional Dynamical Systems in Mechanics and Physics. Springer-Verlag, New York/Berlin.

[39] Constantin, P., \& Foias, C. (1988). Navier-stokes equations. University of Chicago Press.

[40] Gyöngy, I., \& Krylov, N. V. (1981/82). On stochastic equations with respect to seminartingales II. Itô formula in Banach spaces. Stochastics, 6, 153-173.

[41] Liu, K. (2006). Stability of Infinite Dimensional Stochastic Differential Equations with Applications. Chapman and Hall, CRC, London, UK.

[42] Wan, L., \& Duan, J. (2008). Exponential stability of non-autonomous stochastic partial differential equations with finite memory. Statistics \& Probability Letters, 78(5), 490-498.

[43] Kallenberg, O. (2002). Foundations of Modern Probability. Berlin, Springer.

[44] Ash, R. B. (2000). Probability and Measure Theory, Second edition. Academic Press, San Diego.

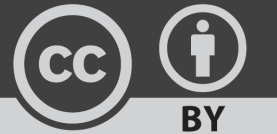

(C) 2020 by the authors; licensee PSRP, Lahore, Pakistan. This article is an open access article distributed under the terms and conditions of the Creative Commons Attribution (CC-BY) license (http://creativecommons.org/licenses/by/4.0/). 